\title{
Early Infant Feeding Practices Among Mothers of Southeast Nigeria
}

\author{
Ihuoma Kathleen Ukpabi ${ }^{1}$, Ebelechuku Francesca Ugochukwu ${ }^{2,}$, , Chinyere Ukamaka Onubogu ${ }^{2}$, \\ Uchenna Ekwochi ${ }^{3}$, Chuka Pius Manyike ${ }^{4}$, Kenneth Nchekwube Okeke ${ }^{2}$ \\ ${ }^{1}$ Department of Paediatrics, Federal Medical Centre, Umuahia, Nigeria \\ ${ }^{2}$ Department of Paediatrics, Nnamdi Azikiwe University, Awka, Nigeria \\ ${ }^{3}$ Department of Paediatrics, Enugu State University of Science and Technology, Enugu, Nigeria \\ ${ }^{4}$ Department of Paediatrics, Ebonyi State University Teaching Hospital, Abakaliki, Nigeria
}

\section{Email address:}

ihuukpabi2014@gmail.com (I. K. Ukpabi),ef.ugochukwu@unizik.edu.ng (E.F.Ugochukwu), cu.onubogu@unizik.edu.ng (C. U. Onubogu), uekwochi@yahoo.co.uk (U. Ekwochi),chukwuka07@yahoo.com (C. P. Manyike),kn.okeke@unizik.edu.ng (K. N. Okeke)

${ }^{*}$ Corresponding author

\section{To cite this article:}

Ihuoma Kathleen Ukpabi, Ebelechuku Francesca Ugochukwu, Chinyere Ukamaka Onubogu, Uchenna Ekwochi, Chuka Pius Manyike, Kenneth Nchekwube Okeke. Early Infant Feeding Practices Among Mothers of Southeast Nigeria. European Journal of Clinical and Biomedical Sciences. Vol. 7, No. 4, 2021, pp. 65-74. doi: 10.11648/j.ejcbs.20210704.13

Received: August 12, 2021; Accepted: August 23, 2021; Published: August 31, 2021

\begin{abstract}
Background: The promotion of optimal infant feeding practices is the most cost-effective of all interventions to reduce infant mortality. In spite of the recommendation of the WHO for optimal breastfeeding practices, several studies in Nigeria on early infant feeding practices have indicated a need for continuous auditing of feeding practices among mothers to make improvements. Objective: This study evaluated the early infant feeding practices of mothers and associated factors. Methods: A cross-sectional, descriptive, multi-centre, questionnaire-based survey was conducted among 2,287 mother-infant pairs in 13 Baby Friendly Hospitals in Southeast Nigeria. Results: The rate of early initiation of breastfeeding [EIBF] within the first one hour of birth was $22.9 \%$, while the exclusive breastfeeding [EBF] rate was $39.3 \%$. The main deterrent to EBF was early introduction of water [94.0\%], formula [40.9\%] and complementary feeds [85.7\%]. There was a statistically significant difference $[p<0.001]$ between the nutritional status of the exclusively breastfed infants and those non-exclusively breastfed, with moderate underweight, and severe underweight malnutrition in the non-exclusively breastfed group. Sociodemographic factors associated with EBF were infant's birth order [ $<<0.001]$, maternal age [ $p=0.010]$, mother's and spouse's education $[p<0.001$ and $p<0.001]$, mother's and spouse's occupation $[p<0.001$ and $p<0.001]$. Conclusion: Policies and measures to promote hospital deliveries targeting all mothers, with special focus on the poor, rural, working, and primiparous mothers, should become a top priority to improve the rate of EIBF. Promotion of EBF with emphasis on the dangers of early introduction of water, formula and complementary feeding should be intensified.
\end{abstract}

Keywords: Early Infant Feeding, Exclusive Breastfeeding, Complementary Feeds

\section{Introduction}

Early infant feeding practices are critical to the survival of newborns and infants [1]. Poor early infant feeding practices compromise the nutritional status and health of infants and have serious implications for their mental and physical development in the critical window from birth to age 2 [2]. The World Health Organization (WHO) recommendations for optimal breastfeeding practices for nursing mothers include early initiation of breastfeeding [EIBF], within one hour of birth, exclusive breastfeeding [EBF], and introduction of nutritionally-adequate and safe complementary [solid] foods at 6 months, together with continued breastfeeding up to 2 years of age or beyond [3]. EIBF within one hour of birth protects the newborn from acquiring infections, hence reducing mortality by $22 \%$ [4]. It prevents hypothermia through skin-to-skin contact, and the resultant colostrum has been appropriately described as 'baby's first immunization' 
[5]. EBF is defined as offering an infant only breast milk, without any other food or drink (not even water) for 6 months of life; allowing only vitamins, minerals and medicines [6]. Optimal breastfeeding practice is the most effective and affordable feeding practice that ensures adequate nutrition in the early months of life with resultant good health indices.

Breastmilk is the infant's natural first food which provides all essential nutrients and immunological factors required for optimal growth and development in the first six months of life [7]. Breastmilk is easily digestible and well absorbed. In addition, EBF reduces deaths from common childhood illnesses - pneumonia and diarrhea [8,9]. Other benefits of EBF include increased bonding between mother and child [10], enhanced cognitive and motor skills in comparison with formula-fed infants [10]. Long-term benefits for mother and child include reduced risk of overweight, obesity and heart diseases in childhood and adolescence, and of developing type 2 diabetes mellitus, breast and ovarian cancers in the mothers $[11,12]$. An exclusively breastfed infant is 14 times less likely to die in the first six months of life than a nonexclusively breastfed child [13]. Approximately $12 \%$ of deaths among under-fives could be averted annually, in lowand medium income countries, if all neonates were exclusively breastfed [14]. Breast milk substitutes are nutritionally inadequate and, if contaminated, increase the risk of infection, with high morbidity and mortality. In subSaharan Africa, approximately $42 \%$ of diarrhea-related deaths among under-fives could be attributed to pre-lacteal feeds or impure water or liquids given to newborns [15]. EBF from birth is possible except for a few medical conditions. Even undernourished mothers can practice EBF, producing a remarkably good quantity and quality of milk [16, 17].

Despite the WHO recommendation and the body of evidence showing the benefits of optimal breastfeeding practices for both mother and infant, early infant feeding practices have remained suboptimal especially in developing countries, with wide variations between and within countries [18]. Globally, only 44\% of infants aged 0-6 months were exclusively breastfed over the period of 2015-2020 [2]. In a meta-analysis of key breastfeeding indicators in 29 subSaharan African countries [19] between 2010-2015, the overall prevalence of EBF ranged from $23.70 \%$ (Central Africa) to $56.57 \%$ (Southern Africa) while the overall prevalence of EIBF within one hour of birth varied between 37.84\% (Central Africa) and 69.31\% (South Africa). West Africa and Central Africa recorded lower overall prevalence of EIBF and EBF than the WHO's recommended target of 50\% [20]. A recent Demographic and Health Survey (DHS) in 13 ECOWAS countries (of which Nigeria is one), between 2010 and 2018, also showed many countries in the region having EBF rates well below the global target $(50 \%)$ required to considerably reduce infant mortality [21, 22]. Although breastfeeding is very common in Nigeria, with $97 \%$ of children ever breastfed, the EBF rate for infants under 6 months was only 29\% [23], while the prevalence of EIBF was $42 \%$ [23]. Within Nigeria, there are regional and state variations in $\mathrm{EBF}$ and $\mathrm{EIBF}$ within one hour of birth rates [23, 24]. Infants living in the Southeast are less likely to be breastfed at 6 months compared to infants living in other areas of the country [25].

The implication of poor early infant breastfeeding practices is their strong correlation with increased burden of infant and child morbidity and mortality [26]. Low prevalence rate of EBF in any region suggests that a huge proportion of newborns within the region are fed water and/or prelacteal foods before the age of at least 6 months [21]. Intake of contaminated prelacteal [21] and complementary foods predispose infants to diarrheal diseases [9]. An estimated 22,371 diarrhea-related deaths among under-fives in Nigeria could have been prevented in 2016 if optimal breastfeeding practices were strictly adhered to by nursing mothers [27]. In Nigeria over $50 \%$ of infants are introduced to solid and semi-solid foods before 6 months, 16\% at 2-3 months, and 40\% are at 4-5 months [28]. Apart from the fact that these foods are often of poor nutritional value, being mostly inadequate in terms of energy, proteins and micronutrients, complementary foods introduced to infants aged less than 6 mo reduce the quantity of breastmilk they consume and could predispose them to early cessation of breastfeeding. Undernutrition is a major consequence of suboptimal breastfeeding practices. This is particularly important in many low and medium-income countries such as Nigeria, with high burden of malnutrition [22, 26].

Breastfeeding practices are determined by various sociodemographic and sociocultural factors which vary between and within countries [21, 29]. Understanding early infant feeding practices of mothers in Southeast Nigeria and the factors that affect these practices are critical to key stakeholders in formulating effective and integrated health policies and interventions. Furthermore, the findings from our study may provide information for evidence-based and cost-effective interventions geared towards improving early infant feeding practices in the Southeast and other regions of Nigeria and subsequently reduce the high burden of infant and child morbidity and mortality. In this study, we therefore examined the early breastfeeding practices of mothers including the rate of EIBF within one hour of birth of infants 0-24 months of age, the EBF rates, the potential sociodemographic factors associated with $\mathrm{EBF}$, and the effect of early infant feeding practices of mothers on weight-for-age $Z$ score of their infants.

\section{Subjects and Methods}

This hospital-based cross-sectional study was conducted across the five states of the South-East geopolitical zone of Nigeria, in 13 health facilities - namely, Nnamdi Azikiwe University Teaching Hospital, Nnewi; Iyienu Hospital, Ogidi; Waterside Specialist Hospital, Onitsha; Federal Medical Centre, Owerri; Ebonyi State University Teaching Hospital, Abakaliki; Enugu State University Teaching Hospital, Enugu; Chukwuemeka Odumegwu Ojukwu University Teaching Hospital, Awka; St. Charles Borromeo Specialist Hospital, Onitsha; Awka-Etiti Catholic Mission Hospital, Awka-Etiti; Immaculate Heart Hospital, Nkpor; St. Patrick's Hospital, 
Enugu; Uwani Health Centre, Enugu and Federal Medical Centre, Umuahia.. The study was conducted over a period of 3 months between July and September, 2018. In each of the states, the major Baby friendly health facilities that offered maternal and child health services were selected. Data was collected from consenting mothers attending well-baby (Immunization) clinics using an interviewer-administered questionnaire consecutively. The Southeast region of Nigeria comprises Anambra, Enugu, Imo, Abia and Ebonyi states. The Igbos are indigenous to this region, even though it is home to other tribes. The dominant religion is Christianity. The region occupies a land mass of $28.98 \mathrm{~m}^{2}$ with a population of 16.39 million people [11.68\% of national population according to 2006 national population census].

Measures

A semi-structured interviewer-administered questionnaire was used. The following socio-demographic characteristics of the subjects were obtained: age in years of mothers, level of education of mother and spouse, occupation of mother and spouse, family size, and mothers' index baby's characteristics.

The early infant (first 6 months) feeding practices of the mothers were assessed with the following measures: early initiation of breastfeeding $[\mathrm{EIBF}]$ by whether or not breastfeeding was initiated within one hour of birth; exclusive breast feeding [EBF] practice or not. Frequency of breastfeeding was assessed by whether or not the mothers offered their infants breastmilk on demand or on a schedule.

Data handling and analysis
Data was analyzed using SPSS version 21. The relationship between two categorical variables or a categorical and an ordinal variable was examined using Chisquare test while Kendall's tau-b was used to test the association between two ordinal variables. Any p-value less than 0.05 was considered statistically significant.

Ethical Considerations

Ethical clearance for the study was obtained from the Research and Ethics Committee (REC) of Nnamdi Azikiwe University Teaching Hospital, Nnewi Anambra State, Southeast Nigeria. Written informed consent was obtained from participants. Permission was duly obtained from all the Clinical Heads of the health facilities used for the study. The consent of the Chief Nursing Officers in charge of the Well Baby Clinics was also obtained.

\section{Results}

Table 1 shows the socio-demographic characteristics of the mother-infant dyads. A total of 2,287 mothers participated in the study. Most of them (93.9\%) were within the age brackets 20-30 years and 30-40 years, with over half (54\%) in the former. The majority (74\%) had a total family size of $\leq 6$. Majority (95.1\%) had at least secondary school education. Among the infants there were slightly more females (50.8\%) than males $(49.2 \%)$, and most $(94.0 \%)$ were born with normal birth weight.

Table 1. Socio-demographic characteristics of mother-baby pairs.

\begin{tabular}{|c|c|c|}
\hline Characteristic & Frequency & Percent \\
\hline \multicolumn{3}{|l|}{ Age of Index Baby } \\
\hline$\leq 6$ months & 849 & 37.1 \\
\hline 7-12 months & 895 & 39.1 \\
\hline$>12$ months & 543 & 23.7 \\
\hline \multicolumn{3}{|l|}{ Sex of Index Baby } \\
\hline Male & 1125 & 49.2 \\
\hline Female & 1162 & 50.8 \\
\hline \multicolumn{3}{|l|}{ Index baby's Birth weight category } \\
\hline Normal birth weight $(\geq 2.5 \mathrm{~kg}$ ) & 2149 & 94.0 \\
\hline Low birth weight $(<2.5 \mathrm{~kg})$ & 110 & 4.8 \\
\hline Not sure & 28 & 1.2 \\
\hline \multicolumn{3}{|l|}{ Family size } \\
\hline$\leq 6$ & 1739 & 76.0 \\
\hline$>6$ & 548 & 24.0 \\
\hline \multicolumn{3}{|l|}{ Participant's age (years) } \\
\hline$<20$ & 75 & 3.3 \\
\hline $20-30$ & 1234 & 54.0 \\
\hline $31-40$ & 913 & 39.9 \\
\hline$>40$ & 65 & 2.8 \\
\hline \multicolumn{3}{|l|}{ Participant's level of education } \\
\hline No formal education & 38 & 1.7 \\
\hline Primary education & 76 & 3.3 \\
\hline Secondary education & 788 & 34.5 \\
\hline Post-secondary education & 1385 & 60.6 \\
\hline Total & 2287 & 100.0 \\
\hline
\end{tabular}

The exclusive breastfeeding practices of the respondents are shown in Table 2. Only $22.9 \%$ of the mothers practiced EIBF within one hour of birth. The practice of EBF was relatively low with $39.3 \%$ (898) respondents feeding their infants only breastmilk during the first six months of their life. However, a sizeable number $(71.5 \%)$ practiced breastfeeding on demand. The major deterrent to EBF was early introduction of water, infant formula and 
complementary feeds (Figure 1 and Table 2). Among those who did not practice exclusive breastfeeding, majority (90.3\%) introduced water along with breastmilk, and $40.9 \%$ introduced formula between birth and 6 weeks. The practice of EBF had a significant positive impact on the nutritional status of the infants (Table 3 ).

Table 2. Exclusive breastfeeding practice.

\begin{tabular}{lll}
\hline Exclusive breastfeeding practice & Frequency & Percent \\
\hline Onset of breastfeeding & & 524 \\
$<1$ hour & 1763 & 22.9 \\
$\geq 1$ hour & & 87.1 \\
Breastfeeding practice within first 6 months & 898 & 39.3 \\
Only breastmilk & 526 & 23.0 \\
Breastmilk and water & 379 & 16.6 \\
Breastmilk and infant formula & 484 & 21.2 \\
Breastmilk and early complementary feeds & & 71.5 \\
Frequency of breastfeeding & 1636 & 28.5 \\
On demand & 651 & 100.0 \\
At regulated times & 2287 & \\
Total & & \\
\hline
\end{tabular}

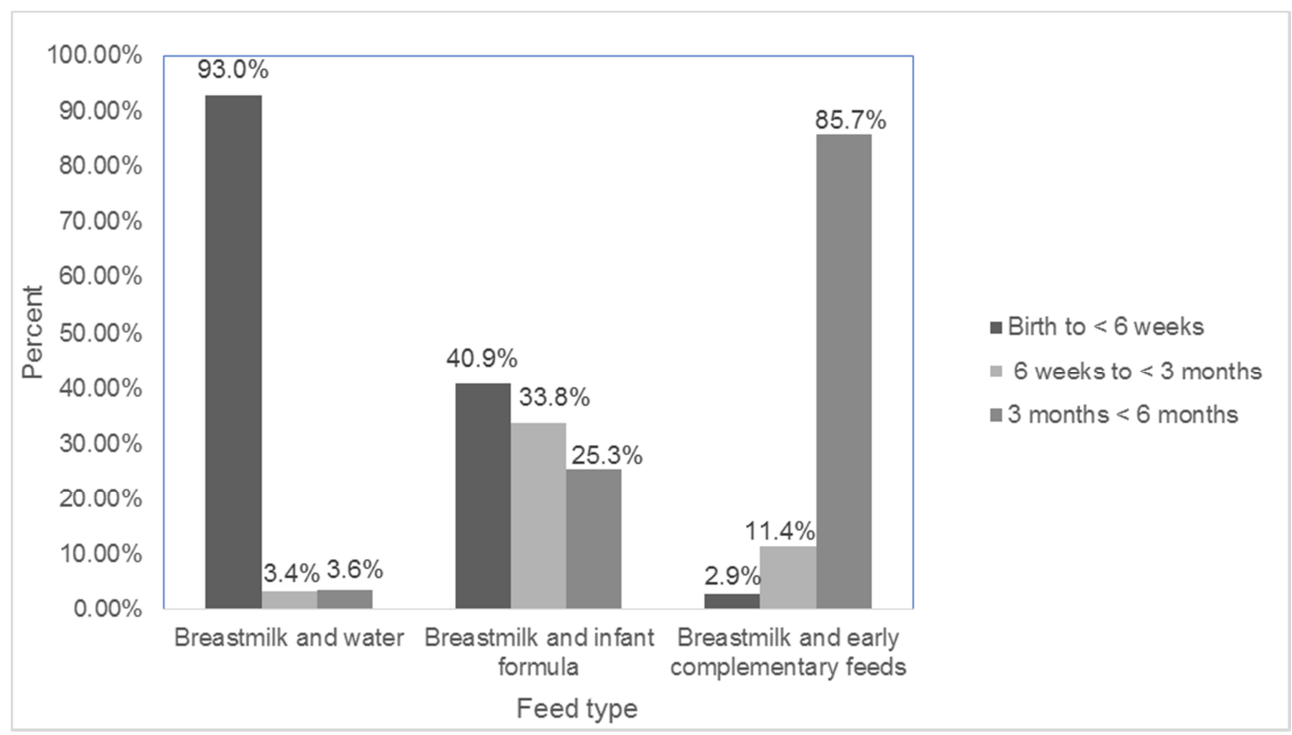

Figure 1. Timing of initiation of other feeds among non-exclusively breastfeeding mothers.

Table 3. Relationship between exclusive breastfeeding pattern and baby's weight for age Z-score [WAZ].

\begin{tabular}{|c|c|c|c|c|c|}
\hline \multirow[b]{2}{*}{$\begin{array}{l}\text { Breastfeeding pattern during early } \\
\text { infancy (within the first } 6 \text { months) }\end{array}$} & \multicolumn{4}{|c|}{ Index Baby's Weight-for-age Z-score } & \multirow[b]{2}{*}{ P-value } \\
\hline & $\begin{array}{l}>-2 \text { to }+2 \mathrm{Z} \text { score } \\
\text { (Normal) }\end{array}$ & $\begin{array}{l}\leq-2 \mathrm{Z} \text { to } \geq-3 \mathrm{Z} \text { score } \\
\text { (Moderate underweight) }\end{array}$ & $\begin{array}{l}\leq-3 \mathrm{Z} \text { score (Severe } \\
\text { underweight) }\end{array}$ & $\begin{array}{l}+2 \mathrm{Z} \text { score } \\
\text { (Overweight) }\end{array}$ & \\
\hline Only breastmilk & $845(94.1)$ & $16(1.8)$ & $5(0.6)$ & $32(3.6)$ & \\
\hline Breastmilk and water & $372(70.7)$ & $101(19.2)$ & $41(7.8)$ & $12(2.3)$ & \\
\hline Breastmilk and infant formula & $301(78.2)$ & $30(7.9)$ & $9(2.4)$ & $42(11.1)$ & $<0.001$ \\
\hline Breastmilk and early complementary feeds & $361(75.5)$ & $80(16.5)$ & $27(5.6)$ & $13(2.7)$ & \\
\hline Total & $1879(82.2)$ & $227(9.9)$ & $82(3.6)$ & $99(4.3)$ & \\
\hline
\end{tabular}

Percentages in parentheses

There was no growth faltering ( $>-2$ to $+2 \mathrm{Z}$ score) during the 6 months of EBF among almost all the infants ((94.1\%) whereas a good proportion of infants who were not exclusively breastfed had moderate $[\leq-2 \mathrm{z}$ score to $>-3 \mathrm{z}$ score $]$ and severe underweight $[\leq-3 \mathrm{Z}$ score $]$ malnutrition with a significant proportion $(16.1 \%)$ in the overweight category $(>+2 \mathrm{Z}$ score). The prevalence rates of moderate and severe underweight malnutrition were highest among infants fed with breastmilk and water, [19.2\% and 7.8\%], followed by those who received breast milk and complementary food [16.5\% and 5.6\%]. Those fed breastmilk and infant formula were more overweight [11.1\%] than the other two groups of non-exclusively breastfed infants. These differences were statistically significant $[\mathrm{p}<0.001]$.

An analysis of the factors associated with the practice of breastfeeding is given in Tables 4 and 5. Maternal age was significantly associated with EBF $(p=0.010)$. Mothers in the age bracket 20-40 years were more likely to practice EBF compared to their counterparts aged 40 and above and younger than 20 years. Educational attainment and occupation of respondents and their spouses were significantly associated $[p<0.001]$ with the practice of EBF 
with a higher proportion of those who had higher education and those employed as professionals or civil/public servants being more likely to practice EBF compared to the less educated and those on lower cadre of employment. The rate of EBF practice among unemployed mothers [32.6\%] was lower than those of civil servants [43.1\%] and even the very busy professionals [39.2\%]. Family size of respondents, sex and birth category of infant did not significantly affect the practice of $\operatorname{EBF} \quad(\mathrm{p}=0.307 ; \mathrm{p}=0.817 ;$ and $\mathrm{p}=0.162$ respectively), but respondents had the trend of decreasing their practice of exclusive breastfeeding with increasing birth order (Table 4 and Table 5), and this was statistically significant $(\mathrm{p}<0.001)$. Relationship between exclusive breastfeeding and the nutritional status of infants is outlined in Table 3.

Table 4. Sociodemographic factors associated with breastfeeding pattern.

\begin{tabular}{|c|c|c|c|c|c|}
\hline Socio-demographic characteristics & Only breastmilk & $\begin{array}{l}\text { Breastmilk and } \\
\text { water }\end{array}$ & $\begin{array}{l}\text { Breastmilk and } \\
\text { infant formula }\end{array}$ & $\begin{array}{l}\text { Breastmilk and } \\
\text { complementary feed }\end{array}$ & P-value \\
\hline \multicolumn{6}{|l|}{ Age (years) } \\
\hline$<20$ & $23(30.7)$ & $23(30.7)$ & $17(22.7)$ & $12(16.0)$ & \multirow{3}{*}{0.010} \\
\hline $31-40$ & $358(39.2)$ & $202(22.1)$ & $169(18.5)$ & $184(20.2)$ & \\
\hline$>40$ & $18(27.7)$ & $11(16.9)$ & $15(23.1)$ & $21(32.3)$ & \\
\hline \multicolumn{6}{|l|}{ Level of Education } \\
\hline Primary education & $32(42.1)$ & $20(26.3)$ & $5(6.6)$ & $19(25.0)$ & \multirow{3}{*}{$<0.001$} \\
\hline Secondary education & $261(33.1)$ & $213(27.0)$ & $110(14.0)$ & $204(25.9)$ & \\
\hline Tertiary & $593(42.8)$ & $283(20.4)$ & $262(18.9)$ & $247(17.8)$ & \\
\hline \multicolumn{6}{|l|}{ Occupation } \\
\hline Professionals & $98(39.2)$ & $52(20.8)$ & $58(23.2)$ & $42(16.8)$ & \multirow{6}{*}{$<0.001$} \\
\hline Civil/public servants & $280(43.1)$ & $129(19.8)$ & $130(20.0)$ & $111(17.1)$ & \\
\hline Traders & $193(36.1)$ & $145(27.1)$ & $82(15.3)$ & $115(21.5)$ & \\
\hline Artisans & $72(36.7)$ & $49((25.0)$ & $27(13.8)$ & $48(24.5)$ & \\
\hline Subsistence farmers & $13(31.7)$ & $9(22.0)$ & $7(17.1)$ & $12(29.3)$ & \\
\hline Unemployed & $218(42.0)$ & $113(21.8)$ & $64(12.3)$ & $124(23.9)$ & \\
\hline \multicolumn{6}{|l|}{ Spouse's occupation } \\
\hline Professional & $145(44.9)$ & $53(16.4)$ & $62(19.2)$ & $63(19.5)$ & \multirow{7}{*}{$<0.001$} \\
\hline Civil/public servant & $266(43.5)$ & $135(22.1)$ & $92(15.0)$ & $119(19.4)$ & \\
\hline Trader & $367(38.6)$ & $230(24.2)$ & $161(16.9)$ & $193(20.3)$ & \\
\hline Artisan & $50(30.3)$ & $51(30.9)$ & $24(14.5)$ & $40(24.2)$ & \\
\hline Driver & $38(27.3)$ & $35(25.2)$ & $24(17.3)$ & $42(30.2)$ & \\
\hline Unskilled worker & $18(33.3)$ & $7(13.0)$ & $14(25.9)$ & $15(27.8)$ & \\
\hline Subsistence farmer/unemployed & $14(32.6)$ & $15(34.9)$ & $2(4.7)$ & $12(27.9)$ & \\
\hline \multicolumn{6}{|l|}{ Spouse's Level of Education } \\
\hline No formal education & $13(28.3)$ & $15(32.6)$ & $7(15.2)$ & $11(23.9)$ & \multirow{4}{*}{$<0.001$} \\
\hline Primary education & $60(39.5)$ & $37(24.3)$ & $19(12.5)$ & $36(23.7)$ & \\
\hline Secondary education & $287(32.7)$ & $242(27.6)$ & $146(16.6)$ & $202(23.0)$ & \\
\hline Tertiary education & $538(44.4)$ & $232(19.1)$ & $207(17.1)$ & $235(19.4)$ & \\
\hline$>6$ & $198(36.1)$ & $135(24.6)$ & $99(18.1)$ & $116(21.2)$ & \multirow{2}{*}{0.307} \\
\hline Total & $898(39.3)$ & $526(23.0)$ & $379(16.6)$ & $484(21.2)$ & \\
\hline
\end{tabular}

Table 5. Sociodemographic factors associated with breastfeeding practice.

\begin{tabular}{|c|c|c|c|c|c|}
\hline \multirow{2}{*}{$\begin{array}{l}\text { Index Infant } \\
\text { characteristics }\end{array}$} & \multicolumn{5}{|c|}{ Breastfeeding Practice } \\
\hline & Only breastmilk & $\begin{array}{l}\text { Breastmilk and } \\
\text { water }\end{array}$ & $\begin{array}{l}\text { Breastmilk and } \\
\text { infant formula }\end{array}$ & $\begin{array}{l}\text { Breastmilk and } \\
\text { complementary feeds }\end{array}$ & P value \\
\hline \multicolumn{6}{|l|}{ Birth order } \\
\hline $1-2$ & $511(42.1)$ & $279(23.0)$ & $185(15.2)$ & $239(19.7)$ & \multirow{4}{*}{$<0.001$} \\
\hline $3-4$ & $332(37.1)$ & $210(23.4)$ & $158(17.6)$ & $196(21.9)$ & \\
\hline $5-6$ & $51(31.7)$ & $37(23.0)$ & $34(21.1)$ & $39(24.2)$ & \\
\hline$>6$ & $4(25.0)$ & $0(0.0)$ & $2(12.5)$ & $10(62.5)$ & \\
\hline \multicolumn{6}{|l|}{ Sex } \\
\hline Male & 447 (39.7) & $264(23.5)$ & $184(16.4)$ & $230(20.4)$ & \multirow{2}{*}{0.817} \\
\hline Female & $451(38.8)$ & $262(22.5)$ & $195(16.8)$ & $254(21.9)$ & \\
\hline \multicolumn{6}{|l|}{ Birth weight category } \\
\hline Low Birth Weight & $36(32.7)$ & $22(20.0)$ & $22(20.0)$ & $30(27.3)$ & \multirow{2}{*}{0.162} \\
\hline Normal & $860(40.0)$ & $501(23.3)$ & $347(16.1)$ & $441(20.5)$ & \\
\hline
\end{tabular}




\section{Discussion}

This multi-center study assessed the early infant feeding practices of mothers in 13 health facilities in Southeast Nigeria. According to WHO, 0-29\% prevalence of EIBF within one hour of birth is considered poor, 30-49\% as fair, $50-89 \%$ as good and $90-100 \%$ as very good [22]. Our study showed a prevalence rate of EIBF within one hour of delivery of $22.9 \%$, which is considered poor. This finding is below the current national prevalence rate of $42 \%$ [23] and the average rate of $37.3 \%-69.3 \%$ obtained for sub-Saharan African countries [19]. Nonetheless, it is comparable to the $19.2 \%, 27.0 \%$, and $23.5 \%$ previously reported in Enugu, South-east [30], Lagos, Southwest [31], and Benin, Southsouth [32] Nigeria, but higher than the $8 \%$ reported in a rural part of Sokoto, North-west [33] Nigeria; and the 3.4\% reported among Nepalese [34] women. It is however lower than the $78.3 \%, 85 \%$, and $48.2 \%$ reported in Ile-Ife, Southwest [35], Nigeria, in a slum area of Bahir Dar Ethiopia [36], and by a previous study in Nnewi, [37] Southeast, Nigeria respectively. Our finding is also consistent with the $18.2 \%$ reported for the Southeast by NNHS in Nigeria [38] and with rates obtained for the individual states in Southeast given as: Abia (21\%), Anambra (16.8\%), Enugu (22.1\%), Ebonyi (19.5\%) and Imo $(3.9 \%)$. This unacceptably low prevalence of EIBF within one hour of birth $(22.9 \%)$ in Southeast Nigeria suggests a decline from the $37.2 \%$ obtained from an aggregated data for geopolitical zones over a 5 year-period reported in 2013 [39] for the zone, and the $48.2 \%$ obtained by the previous Nnewi [37] study in the same zone and, indicates that proactive interventions should be embarked upon by all stakeholders to forestall further decline, and rather ensure a rise. Place of delivery, place of residence (rural or urban), cultural, maternal health factors at the time of delivery, maternal age at birth of the index child, maternal educational attainment, and mode of delivery are some of the factors reported to be associated with timing of breastfeeding initiation $[19,23,36,38]$. The Ethiopian model is worthy of emulation. The EIBF of $85 \%$ [36] indicated an increase compared to a previous study within the region. This was attributed to the provision of free delivery services in the country, with persistent promotion of same, resulting in a dramatic increase in the number of mothers who gave birth in health institutions, creating a good opportunity for health professionals to promote the initiation of breastfeeding within one hour of birth.

The $39.3 \%$ prevalence rate of exclusive breast feeding obtained in this study is consistent with the $37.3 \%$ obtained by a previous study in Nnewi, South-east [37], 40.7\% in Benin City, South-south [40], and $41.4 \%$ in Lagos, Southwest [41], Nigeria. This is, however, low compared to the global target of $50 \%$ by 2025 [20], and reports from other studies $[33,42]$ although it suggests an improvement on the $15.8 \%-29.0 \%$ reported for the Southeast [23] by NDHS 2018. Prevalence rates lower than our finding have also been reported within and outside Nigeria [17, 43]. The fact that low prevalence of EBF is strongly correlated with increased incidence of childhood morbidity and mortality has been severally reported $[12,15,18,27]$. It stands to reason, therefore, that more effort should be made towards improving the practice of EBF in Nigeria. It has been estimated that approximately $12 \%$ of deaths among children under five years of age could be averted annually in low- and medium-income countries if all neonates were exclusively breastfed [17]. The wide variations in the prevalence rates of EBF observed within the same country and between countries and regions may be multifactorial in origin. Sociodemographic and sociocultural factors are equally dynamic, varying from community to community and between countries, including whether the study setting is urban or rural.

A large proportion of mothers $(71.5 \%)$ in the study, practiced breastfeeding of their infants on demand. This is comparable to the report of a previous study carried out in Enugu [44] (63.5\%) but lower than the findings of previous studies in Nnewi [37] $(92.5 \%)$ and Owerri urban [45] $(93.8 \%)$, all in southeast Nigeria. Breastfeeding infants on demand results in ample breastmilk production and adequate nutrition for the infant, unlike breastfeeding at fixed schedules.

The major deterrents to EBF were early introduction of water, infant formula and complementary feeds. Several studies have similarly reported the practice of early introduction of water, infant formula and complementary feeds $[27,37,39,40,41,44]$. However, the rates we found in this study are higher than some reported national rates [28]. Early introduction of water, infant formula and complementary feeds, which may even be contaminated, translates to the infant taking less of the nutritious breastmilk, and these practices have been associated with increased incidence of under-nutrition, diarrhea, reduced lactation or even early cessation of breastfeeding and consequently, increased infant mortality [12, 15, 18, 27, 37] The finding of a statistically significant difference between the nutritional status of the exclusively breastfed infants and the non-exclusively breastfed group, with a good proportion of the non-exclusively breastfed infants having significant moderate and severe undernutrition, substantiates these reports.

The significant association between respondents' age and the practice of EBF is consistent with the report of Agho et al [21] in a recent review of EBF practices in 13 ECOWAS countries (including Nigeria). This showed that higher maternal age (35-49 years) was associated with increased odds against EBF compared to a younger age (15-19 years). A Brazilian study [46] reported that adolescent mothers had a 1.5 times greater risk of abandoning EBF before their babies were six months of age when compared with adult women. Factors that have been associated with poor practice of EBF among this age include low socioeconomic status, fatigue, back pain during breastfeeding, breast engorgement, sore nipples and mastitis, and negative familial influence [21, 47]. 
The positive association between the practice of EBF and maternal education in this study validates the findings of other studies [21, 24, 37, 40, 44, 48, 49]. This finding is not surprising considering that education emancipates the mind from the stranglehold of contrary traditional beliefs and cultures regarding child feeding practices. Educated mothers are equally more likely to gain clearer understanding of health talks and sensitization messages given by health workers during antenatal visits, labour and delivery, postpartum and postnatal visits, and may also have more access to health information promoting optimal breastfeeding practices. The finding however contrasts with other reports $[33,50]$ which stated that mothers who were unable to read and write or in primary school were 3 times more likely to practice EBF than those who completed secondary school. The explanation given for this finding was that the better educated women could have better opportunity for employment that could lead them out of city for the whole day.

Our study showed that the effect of paternal education on the practice of EBF was highly significant and this is comparable to the reports of studies done in Enugu, Southeast, Nigeria [30], Tanzania [51] and Dhaka city, Bangladesh [52]. The possible reason for this is that education of fathers and mothers has a very strong impact on perception of breastfeeding. It has been shown that teamwork parenting could increase the success of EBF [52, 53] and a RCT research in Canada [54] conveyed that collaboration between parents influenced their knowledge, perceptions, practices, and duration of EBF. However, some studies have indicated that there is no association between paternal education and practice of EBF [40, 55].

There was a significant association between the practice of EBF and occupation of both mother and spouse with a higher proportion of professionals, highly placed civil and public servants being more inclined to practice EBF. This is similar to the finding in Enugu [44], which is not surprising considering the fact that professionals and highly placed public servants are highly educated people who easily understand the benefits of EBF. Where there is understanding, strategies to surmount the constraints, such as expressing breastmilk, either manually or with breast pumps, so that someone else could feed the infant with the mother's milk while she is at work could be employed. In addition, the report of Society for Human Resource Management, 2019 survey indicates that $51.0 \%$ of employers in Nigeria have worksite/on-site lactation support programs including on-site lactation/mother's room which is positive for EBF in Nigeria. Flexible work schedules and proximity to work places can help sustain EBF for these working mothers and fathers. This finding is however at variance with a previous study in Indonesia [49] which reported that unemployed mothers had better EBF practice.

The finding in our study that practice of EBF was associated with birth order, with higher EBF rates for the first 1-2 children than subsequent children, is comparable with the report from Port Harcourt, South-south [56] and Abakaliki,
Southeast [57] Nigeria. A probable reason could be because of the great enthusiasm and family support that greet the birth of the first and the second children in Nigeria. Our finding, however, contrasts with the report of a previous study done in Enugu, Southeast, Nigeria [30] which stated that infants of first birth order had $25 \%$ less likelihood of being exclusively breastfed compared with second and higher birth orders. It is pertinent to note that although breastfeeding practices and experiences are culture dependent, they are equally context bound. Situations whereby some grandmothers and mothersin-law who have come to provide family support unexpectedly play roles that interfere with the role of the mother as the primary care-giver of the infant have been documented [48]. Lack of family support and encouragement in addition to the inexperience of younger mothers, especially those having difficulties with establishing breastfeeding have been associated with low EBF rates [21, 48].

\section{Conclusions}

The practice of EIBF within one hour of birth is poor $(22.9 \%)$ among mothers in Southeast Nigeria. Urgent measures by all stake holders to promote hospital deliveries, targeting promotion of EIBF with special focus on poor, rural, working, and primiparous mothers should become a top priority.

Barely a third of mothers practiced EBF, the major deterrent being early introduction of water, infant formula and complementary feeds. Nutrition education at the community level should be embarked upon as a top priority by the Local Health Authorities in this zone.

\section{Limitation of the Study}

Recall bias may have been a limitation of the study as mothers had to recall their infant feeding practices. Another limitation is that this was a cross sectional study which is unable to demonstrate causality.

\section{Conflict of Interest}

All authors declare no conflict of interest.

\section{Ethics}

Ethical clearance for the study was obtained from the Research and Ethics Committee (REC) of Nnamdi Azikiwe University Teaching Hospital, Nnewi Anambra State, Southeast Nigeria. The research was carried out in accordance with the Declaration of Helsinki.

\section{Acknowledgements}

We are grateful to the nurses in the health facilities used for the study for their assistance in organizing the participants during of the study. Our thanks also go to the house officers who assisted with data entry. 


\section{References}

[1] World Health Organization. Exclusive breastfeeding for optimal growth, development and health of infants 2018. Available from https://www.who.int/elena/titles/exclusive_breastfeeding/en/.

[2] World Health Organization. Infant and young child feeding August 2020. Available from: https://www.who.int/newsroom/fact-sheets/detail/infant-and-young-child-feeding.

[3] Kramer MS, Kakuma R. The Optimal Duration of Exclusive Breastfeeding: A systematic Review. Adv Exp Med Biol 2004; 554: 63-77. doi: 10.1007/978-1-4757-4242-8_7. PMID: 15384567.

[4] Edmond KM, Zandoh C, Quigley MA, Amenga-Etego S, OwusuAgyei S, Kirkwood BR. Delayed breastfeeding initiation increases risk of neonatal mortality. Pediatrics. 2006; 117 (3): e380-6. doi: 10.1542/peds.2005-1496. PMID: 16510618.

[5] Sharma IK, Byrne A, Early initiation of breastfeeding: a systematic review of the factors and barriers in South Asia. Int Breastfeed J. 2016; 11: 17.

[6] World Health Organization. Indicators for Assessing Infant and Young Child Feeding Practices 1; World Health Organization: Geneva, Switzerland, 2008.

[7] Ballard O, Morrow AL. Human milk composition: nutrients and bioactive factors. Pediatric Clinics North America. 2013: 60 (1): 49-74.

[8] Kramer MS, Chalmers B, Hodnett ED, Sevkovskaya Z, Dzikovich I, Shapiro S, et al; PROBIT Study Group (Promotion of Breastfeeding Intervention Trial). Promotion of Breastfeeding Intervention Trial (PROBIT): a randomized trial in the Republic of Belarus. JAMA. 2001 Jan 24-31; 285 (4): 413-20. doi: 10.1001/jama.285.4.413. PMID: 11242425.

[9] Ogbo FA, Agho K, Ogeleka P, Woolfenden S, Page A, Eastwood J; Global Child Health Research Interest Group. Infant feeding practices and diarrhoea in sub-Saharan African countries with high diarrhoea mortality. PLoS One. 2017 Feb 13; 12 (2): e0171792. doi: 10.1371/journal.pone.0171792. PMID: 28192518; PMCID: PMC5305225.

[10] Black RE, Allen LH, Bhutta ZA, Caulfield LE, de Onis M, Ezzati M, Mathers C, Rivera J; Maternal and Child Undernutrition Study Group. Maternal and child undernutrition: global and regional exposures and health consequences. Lancet. 2008 Jan 19; 371 (9608): 243-60. doi: 10.1016/S0140-6736(07)61690-0. PMID: 18207566.

[11] Victora CG, Bahl R, Barros AJ, França GV, Horton S, Krasevec J, et al; Lancet Breastfeeding Series Group. Breastfeeding in the 21st century: epidemiology, mechanisms, and lifelong effect. Lancet. 2016 Jan 30; 387 (10017): 475-90. doi: 10.1016/S0140-6736(15)01024-7. PMID: 26869575.

[12] Mannan H. Early Infant Feeding of Formular or Solid Foods and Risk of Childhood Overweight or Obesity in a Socioeconomically Disadvantaged Region of Australia: A Longitudinal Cohort Analysis. Int. J. Environ. Res. Public Health. 2018; 15: 1685.

[13] Loaiza E, Wardlaw T, Salama P. Child mortality 30 years after the Alma-Ata Declaration. The Lancet 2008; 372 (9642): 874-876. Doi: https://doi.org/10.1016/S0140-6736(08)61378-1.
[14] Debes AK, Kohli A, Walker N, Edmond K, Mullany LC. Time to initiation of breastfeeding and neonatal mortality and morbidity: a systematic review. BMC Public Health. 2013; 13 Suppl 3 (Suppl 3): S19. doi: 10.1186/1471-245813-S3-S19. Epub 2013 Sep 17. PMID: 24564770; PMCID: PMC3847227.

[15] Wardlaw T, Salama P, Brocklehurst C, Chopra M, Mason E. Diarrhoea: why children are still dying and what can be done. Lancet. 2010 Mar 13; 375 (9718): 870-2. doi: 10.1016/S01406736(09)61798-0. Epub 2009 Oct 14. PMID: 19833382.

[16] Tella A, Falaye A, Aremu O. Hospital-based assessment of breast feeding, behaviour and practices among nursing mothers in Nigeria and Ghana. Pak J Nutr. 2014; 7: 165-171.

[17] Venuta A, Vaienti F. Children's feeding practices in families from Mahgreb living in Italy. The Internet Journal of Nutrition and Wellness. 2013: 2 [2]: 34

[18] Ogbo FA, Page A, Idoko J. et al. Have policy responses in Nigeria resulted in improvements in infant and young child feeding practices in Nigeria? Int. Breastfeed. J. 2017: 12: 9.

[19] Issaka AI, Agho KE, Renzaho AMN. Prevalence of key breastfeeding indicators in 29 sub-Saharan African countries: a meta-analysis of demographic and health surveys (20102015). BMJ Open, 2017; 7 (10): e014145. doi.1136/bmjopen2016-014145.

[20] World Health Organization. Global Nutrition Targets 2025: Policy Brief Series (WHO/NMH/HND/14.2); World Health Organization: Geneva, Switzerland, 2014. Available at: https://www.who.int/nutrition/publications/globaltargets2025 policybrief_overview/en/.

[21] Agho KE, Ezeh OK, Ghimire PR. et al. Exclusive Breastfeeding Rates and Associated Factors in 13 'Economic Community of West African States" (ECOWAS) Countries. Nutrients. 2019: 11 (12): 3007.

[22] World Health Organization. Infant and Young Child Feeding: A tool for assessing national practices, policies and programmes. Available https://apps.who.int/iris/handle/10665/42794.

[23] National Population Commission (NPC) [Nigeria] and ICF. Nigeria Demographic and Health Survey 2018 Key Indicators Report. Abuja, Nigeria, and Rockville, Maryland, USA: NPC and ICF, 2019. Available at: https://dhsprogram.com/publications/publication-fr359-dhsfinal-reports.cfm.

[24] Adewuyi EO, Adefemi K. Breastfeeding in Nigeria: a systematic review. Int J Community Med Public Health 2016 Feb; 3 (2): 385-396.

[25] Centre for Disease Control and Prevention. National Immunization Survey (NIS) 2018-2019: Facts About Nationwide Breastfeeding Goals - Key Breastfeeding Indicators of Infants Born in 2017 Available from https://www.cdc.gov/breastfeeding/data/facts.html.

[26] Black RE, Victora CG, Walker SP, Bhutta ZA, Christian P, de Onis M, et al; Maternal and Child Nutrition Study Group. Maternal and child undernutrition and overweight in lowincome and middle-income countries. Lancet. 2013 Aug 3; 382 (9890): 427-451. doi: 10.1016/S0140-6736(13)60937-X. Epub 2013 Jun 6. Erratum in: Lancet. 2013. 2013 Aug 3; 382 (9890): 396. PMID: 23746772. 
[27] Ogbo FA, Okoro A, Olusanya BO, Olusanya J, Ifegwu IK, Awosemo AO, et al. Diarrhoea deaths and disability-adjusted life years attributable to suboptimal breastfeeding practices in Nigeria: findings from the global burden of disease study 2016 Int Breastfeed J 2019; 14: 4. Available at: https://doi.org/10.1186/s13006-019-0198-9.

[28] National Population Commission (NPC) [Nigeria] and ICF. 2019. 11.2.5 Introduction of Complementary Foods.

[29] Agunbiade OM, Ogunleye OV. Constraints to exclusive breastfeeding practice among breastfeeding mothers in Southwest Nigeria: implications for scaling up. Int Breastfeed J. 2012 Apr 23; 7: 5. doi: 10.1186/1746-4358-7-5. PMID: 22524566 ; PMCID: PMC3359265.

[30] Aghaji MN. Exclusive breast-feeding practice and associated factors in Enugu, Nigeria. West Afr J Med. Jan-Mar; 21 (1): 66-9. PMID: 12081350.

[31] Sebanjo IO, Oshikoya KA, Ogbera OA, Wright KO, Anga Al. Breastfeeding policy and practices at general Paediatric outpatient clinic of a teaching hospital in Lagos, Nigeria. Int Breastfeed J 2014; 9: 10.

[32] Sadoh AE, Sadoh WE, Oniyelu P. Breast feeding practices among medical women in Nigeria. Niger Med J 2011; 52 (1): $7-12$.

[33] Oche MO, Umar AS. Breastfeeding practices of mothers in a rural community of Sokoto, Nigeria. Nigerian Postgraduate Medical Journal 2008; June; 15 (2): 101-104.

[34] Mullany LC, Katz J, Li YM, Khatry SK, LeClerq SC, Darmstadt GL, Tielsch JM. Breast-feeding patterns, time to initiation, and mortality risk among newborns in southern Nepal. J Nutr. 2008 Mar; 138 (3): 599-603. doi: 10.1093/jn/138.3.599. PMID: 18287373; PMCID: PMC2366167.

[35] Mbada CE Olowokere AE, Faronbi JO, Faremi FA, Oginni MO. Breastfeeding profile and practice of Nigerian mothers: a cross-sectional survey. Int $\mathrm{J}$ Reprod Contracept Obstet Gynecol 2014; 3: 969-976.

[36] Demilew YM, Tafere TE, Abitew DB. Infant and young child feeding practice among mothers with 0-24 months old children in a slum area of Bahir Dar City, Ethiopia. Int Breastfeed J. 2017; 12: 26.

[37] Ukegbu AU, Ebenebe EU, Ukegbu PO, Onyeonoro UU. Determinants of breastfeeding pattern among nursing mothers in Anambra State, Nigeria. East Afr J Public Health. 2011 Sep; 8 (3): 226-31. PMID: 23120962.

[38] National Nutrition Study and Health Survey (NNHS) June 2018: Saving One Million Lives Programme for Results. Available at: https://nigerianstat.gov.ng

[39] Yahya WB, Adebayo SA. Modelling the trend and Determinants of Breastfeeding Initiation in Nigeria. Child Dev. Research. 2013; 530396. Available from: https:// doi.org/10.1155/2013/530396.

[40] Akpan UJ, Ibadin MO, Abiodun PO. Breastfeeding practices in early infancy in Benin City, Nigeria. Niger J Paed. 2015; 42 (2): 126-31.

[41] Akinyinka MR, Olatona FA, Oluwole EO. Breastfeeding Knowledge and Practices among mothers of children under 2 years of age living in a military barrack in Southwest, Nigeria.
Int $\mathrm{J}$ of MCH and AIDS 2016; 5 (1); 1-13.

[42] Ojofeitimi EO, Esimai OA, Owolabi OO, Oluwabusi, Olaobaju OF, Olanuga TO. Breastfeeding practices in urban and rural health centres: Impact of Baby Friendly Hospital Initiative in Ile-Ife, Nigeria. Nutrition and Health 2000; 14 (2): 119-125.

[43] Peterside O, Kunle-Oluwu OE, Duru CO. Knowledge and Practice of Exclusive Breast Feeding Among Mothers in Gbarantoru Community, Bayelsa State, Nigeria. IOSR Journal of Dental and Medical Sciences (IOSR-JDMS) 2013; e-ISSN: 2279-0853, p-ISSN: 2279-0861. 12 (6): 34-40.

[44] Anoshirike CO, Ejeogo CP, Nwosu OIC, Maduforo AN, Nnoka KO. Infant Feeding Practices Among Mothers and Their Infants Attending Maternal and Child Health in Enugu, Nigeria. Journal of Biology, Agriculture and Healthcare 2014; 4 (10): 130-139.

[45] Anoshirike CO, Asinobi CO. Associations between caregivers caring behaviour and child's anthropometric status, appetite and food consumption practices in Owerri Municipality. Nig. J of Nutr. Sciences. 2008; 29 (1) 96-110.

[46] Santo LC, de Oliveira LD, Giugliani ER. Factors associated with low incidence of exclusive breastfeeding for the first 6 months. Birth 2007; 34 (3): 212-219. Doi: 10.1111/j.1523536X.2007.00173. x. PMID: 17718871.

[47] De Oliveira LD, Giugliani ERJ, Santo LC, Nunes LM. Counselling sessions increased duration of exclusive breastfeeding: a randomized clinical trial with adolescent mothers and grandmothers. Nutrition Journal 2014; 13: 73-79. Doi: https://doi.org/10.1186/1475-2891-13-73.

[48] Akadri A, Odelola O. Breastfeeding practices among Mothers in Southwest Nigeria. Ethiop J Health Sci. 2020; 30 (5): 697. doi: http://dx.doi.org/10.4314/ejhs.v30i5.8.

[49] Laksono AD, Wulandari RD, Ibad M, Kusrini I. The effects of mother's education on achieving exclusive breastfeeding in Indonesia. BMC Public Health. 2021 Jan 6; 21 (1): 14. doi: 10.1186/s12889-020-10018-7. PMID: 33402139; PMCID: PMC7786474.

[50] Dykes F, Flacking R. Encouraging breastfeeding: A relational perspective. Early Human Development. 2010; 86 (11): 733736.

[51] Nkala TE, Msuya SE. Prevalence and predictors of exclusive breastfeeding among women in Kigoma region, Western Tanzania: a community based cross-sectional study. Int Breastfeed J. 2011; 6: 17-23.

[52] Banu B, Khanom K. Effects of Education Level of Father and Mother on Perceptions of Breastfeeding. J Enam Med Col. 2012; 2 (2): 67-73.

[53] Rempel JK, Rempel LA, Hoa DTP, Vui LT, Long TK. Parenting Teamwork: The Impact of a Fathering Intervention on Mothers and Infants in Vietnam. Child Dev. 2020 Mar; 91 (2): e345-e364. doi: 10.1111/cdev.13244. Epub 2019 Apr 1. PMID: 30937897.

[54] Abbass-Dick J, Stern SB, Nelson LE, Watson W, Dennis CL. Coparenting breastfeeding support and exclusive breastfeeding: a randomized controlled trial. Pediatrics. 2015 Jan; 135 (1): 102-10. doi: 10.1542/peds.2014-1416. Epub 2014 Dec 1. PMID: 25452653. 
[55] Atimati AO, Adam VY. Breastfeeding practices among mothers of children aged 0-24 months in Egor Local Government Area of Edo State, Nigeria. South African Journal of Clinical Nutrition 2020; 33 (10: 10-16. doi: 10. 1080/16070658,2018.1493071.

[56] West BA, Onubogu UC, Okari TG, Aitafo JE. Prevalence, Knowledge, Practice and Problems Associated with Breastfeeding among Mothers/Caregivers Attending a Paediatric Out-Patient Clinic in Port Harcourt, Nigeria. IOSR
Journal of Dental and Medical Sciences (IOSR-JDMS). 2020 May; 19 (5): 20-27. Doi: 10.9790/0853-1905112027.

[57] Anyanwu OU, Ezeonu CT, Ezeanosike OB, Okike CO. The practice of breastfeeding by health care workers in the Federal Teaching Hospital, Abakaliki, Southeastern Nigeria. S Afr J Child Health 2014; 8 (2): 55-58. Doi: 10.7196/sajch.668. 